\title{
Acute effects of cardiac resynchronization therapy on arterial distensibility and serum norepinephrine levels in advanced heart failure
}

\author{
Mustafa Yildiz ${ }^{1}$, Hakan Hasdemir², Ceyhan Turkkan², Mehmet Ali Astarcioglu ${ }^{3}$, \\ Ahmet Taha Alper ${ }^{2}$, Alparslan Sahin ${ }^{4}$, Mehmet Ozkan ${ }^{3}$ \\ ${ }^{1}$ Department of Cardiology, Kartal Koşuyolu Yüksek Ihtisas Educational and Research Hospital, \\ Istanbul, Turkey (Formerly institute of author: Sakarya University) \\ ${ }^{2}$ Department of Cardiology, Dr. Siyami Ersek Cardiovascular and Thoracic Surgery \\ Training and Research Hospital, Istanbul, Turkey \\ ${ }^{3}$ Department of Cardiology, Kartal Koşuyolu Yüksek Ihtisas Educational \\ and Research Hospital, Istanbul, Turkey
}

${ }^{4}$ Department of Cardiology, Bakirkoy Dr. Sadi Konuk Educational and Research Hospital, Istanbul, Turkey

\begin{abstract}
Background: Cardiac resynchronization therapy (CRT) has become an accepted method for treating refractory heart failure $(H F)$. Arterial distensibility is an index of arterial stiffness and a surrogate marker for atherosclerosis. The present study aims to assess the acute effects of ventricular resynchronization therapy with biventricular stimulation on arterial distensibility, echocardiographic parameters and serum norepinephrine levels in patients with drug refractory $H F$.
\end{abstract}

Methods: Fourteen cardiac HF patients (53.6 \pm 9.1; 39-67 years, 7 woman) were enrolled for CRT. Patients had an advanced cardiac HF (NYHA III-IV functional class) due to non-ischemic dilated cardiomyopathy, with a left ventricular ejection fraction (LVEF) $<35 \%$ and QRS duration $\geq 120 \mathrm{~ms}$. Blood samples for norepinephrine and B-type natriuretic peptide were collected before $24 \mathrm{~h}$ biventricular implantation and after $48 \mathrm{~h}$ of CRT. Transthoracic echocardiography was used to evaluate arterial distensibility and cardiovascular condition.

Results: Although systolic blood pressure, diastolic blood pressure, LV end-diastolic diameter, $L V$ end-systolic diameter, serum B-type natriuretic peptide, and serum norepinephrine levels significantly decreased after CRT implantation; EF and aortic distensibility significantly increased $(p<0.05)$. There was no significance in the hemodynamic and echocardiographic values, norepinephrine and B-type natriuretic peptide levels in pre-and post-CRT between man and woman.

Conclusions: The major findings of this study are that in patients with cardiac HF in acute period, after implantation of CRT serum norepinephrine levels decrease and the arterial distensibility improves. (Cardiol J 2013; 20, 3: 304-309)

Key words: cardiac resynchronization therapy, arterial distensibility, norepinephrine, sympathetic activity, B-type natriuretic peptide, heart failure

Address for correspondence: Dr. Mustafa Yildiz, MD, PhD, Prof. Cardiologist, Internal Medicine Specialist and Physiologist, Bayar Cad Gülbahar Sok Emniyet Sitesi D6, Kozyatağı, İstanbul, tel: +90 0532371 17 01, e-mail: mustafayilldiz@yahoo.com Received: 21.10.2012 Accepted: 25.11.2012 


\section{Introduction}

Cardiac resynchronization therapy (CRT) has become an accepted method for treating refractory heart failure (HF) in patients with idiopathic or ischemic dilated cardiomyopathy associated with electromechanical asynchrony. In the current American Heart Association (AHA) guidelines, CRT is a class I (level of evidence A) therapy for patients with a left ventricular ejection fraction (LVEF) less than or equal to $35 \%$ and a QRS duration greater than or equal to $120 \mathrm{~ms}$, who are New York Heart Association (NYHA) functional Class III or IV despite optimal recommended medical therapy $[1,2]$. By restoring co-ordinated contraction, CRT promotes $\mathrm{LV}$ reverse remodeling and improves symptoms, exercise capacity and quality of life in patients with cardiac HF (CHF) $[1,2]$. Several animal studies and clinical trials have suggested that down-regulation of inflammatory cytokines may improve cardiac performance [3].

Arterial distensibility and arterial compliance are an index of arterial stiffness and a surrogate marker for coronary atherosclerosis [4]. Arterial distensibility plays a role in determining both arterial systolic and diastolic pressure and therefore, in the clinical context, influences LV size and function, and coronary blood flow [5, 6]. Non-invasive ultrasound techniques such as echocardiography and aortic pulse wave velocity are used to evaluate arterial distensibility and cardiovascular condition [4-6].

The effect of CRT on arterial distensibility, compliance and inflammation alterations associated with CHF is not well understood [7-9]. Accordingly, the present study aims to assess the acute effects of ventricular resynchronization therapy with biventricular stimulation on arterial distensibility, echocardiographic parameters and serum norepinephrine (NE) levels in patients with symptomatic systolic HF and prolonged QRS duration.

\section{Methods}

\section{Study population and protocol}

In this prospective randomized study, we enrolled $14 \mathrm{CHF}$ patients $(53.6 \pm 9.1 ; 39-67$ years, 7 woman) referred to our institute from January 2010 to January 2011 for biventricular pacemaker implantation. Patients had an advanced CHF (NYHA III-IV functional class) due to non-ischemic dilated cardiomyopathy, with a LVEF $<35 \%$ and QRS duration $\geq 120 \mathrm{~ms}$. All patients had been receiving optimal medical therapy for at least 3 months prior to enrolment. Exclusion criteria were previous pacemaker implantation, significant valvular heart disease requiring surgical correction, acute myocarditis and/or pericarditis and severe chronic obstructive pulmonary disease. Patients had no clinical evidence of inflammatory, neoplastic, or metabolic diseases as assessed by clinical history, examination and laboratory tests.

The study was approved by the local ethical committee. All subjects gave their consent for inclusion in the study. The investigation conforms with the principles outlined in the Declaration of Helsinki.

\section{Blood pressure measurement}

In each subject the arterialblood pressure was measured by the same observer in each subject in the supine position after at least $30 \mathrm{~min}$ of rest. Clinic blood pressure was measured, using a mercury sphygmomanometer with a cuff appropriate to the arm circumference (Korotkoff phase I for systolic blood pressure and $\mathrm{V}$ for diastolic blood pressure). In each subject two blood pressure measurements were performed, and their mean was considered for analysis.

\section{Laboratory measurements}

Blood samples were taken between 08.30 and 09.30 a.m. from the antecubital vein which had fasted overnight. Fasting serum blood glucose, blood urea nitrogen, creatinine, uric acid, aspartat aminotransferase, alanin aminotransferase, total cholesterol, high-density lipoprotein cholesterol, very low-density cholesterol, triglyceride, sodium, potassium, chlorine, calcium and magnesium were measured using an Abbott C8000 (Abbott, Japan) automatic analyzer. Blood cells were counted on the HMX (Beckman Coulter, USA) analyzer. Thyroid stimulating hormone (TSH), free T3 and free T4 were measured with Immulite 2000 (DPC; LosAngeles, USA) by chemiluminescent immunometric assay.

\section{Measurement of norepinephrine and brain natriuretic peptide}

Blood samples for measure basal serum NE and B-type natriuretic peptide (BNP) were collected before $24 \mathrm{~h}$ biventricular implantation and after $48 \mathrm{~h}$ of CRT. All samples were centrifuged at 2000 $\mathrm{rpm}$ for $15 \mathrm{~min}$ and stored at $-80^{\circ} \mathrm{C}$ until further analysis. Plasma levels of BNP (ADVIA Centaur BNP Assay, USA) was measured using a competitive enzyme immunoassay. $\mathrm{NE}\left(\mathrm{DRG}^{\circledR}\right.$ Noradrenaline ELISA, EIA-4317, USA) levels were determined by enzyme-linked immunosorbent assay (ELISA). 


\section{Transthoracic echocardiography}

A Vivid 3 cardiovascular ultrasound system [3S sector probe (1.5-3.6 MHz), GE] was used for transthoracic echocardiographic evaluation [10]. Transthoracic echocardiography was performed before $24 \mathrm{~h}$ biventricular implantation and after $48 \mathrm{~h}$ of CRT. Echocardiography was performed with the subject in the lateral decubitus position. LV end-diastolic diameter, LV end-systolic diameter and LVEF\% were measured in the parasternal long-axis view. The measurements were obtained from two-dimensional guided $\mathrm{M}$-mode recordings. After the routine conventional echocardiographic examination was performed, subjects were placed in a mild recumbent position, and the ascending aorta was recorded in the two-dimensional guided $\mathrm{M}$-mode tracings. Aortic diameters were recorded $3 \mathrm{~cm}$ above the aortic valve by M-mode echocardiography. Aortic systolic diameter was determined at the time of the full opening of the aortic valve, and aortic diastolic diameter was determined at peak QRS. The same blinded investigator performed the echocardiography and the echocardiograms were analyzed by two blinded cardiologists.

Aortic strain was calculated as follows: Aortic strain $=(\mathrm{AoS}-\mathrm{AoD}) / \mathrm{AoD}$, where $\mathrm{AoS}-$ systolic aortic diameter, $\mathrm{AoD}$ - diastolic aortic diameter). Aortic distensibility was calculated as follows: Aortic distensibility $=2 \times(\mathrm{AoS}-\mathrm{AoD}) /(\mathrm{AoD} X \mathrm{PP})$, where $\mathrm{PP}$ - pulse pressure $[11,12]$.

\section{Pacemaker implantation}

In all patients ventricular tachycardia was not induced during elecrophysiological study. Biventricular devices were implanted as previously described [13]. Briefly, the LV pacing lead was inserted transvenously via the subclavian vein with the help of a guiding catheter into the coronary sinus, and then positioned in the lateral or posterolateral cardiac vein when possible.

\section{Statistical analysis}

Statistics were obtained using the ready-to-use programme of SPSS version 8.0. All the values were expressed as mean \pm standard deviation. Wilcoxon's nonparametric test was used for comparison of preand post-CRT factors. The factors between men and women were assessed by Mann-Whitney U Test. $\mathrm{p}<0.05$ was considered significant.

\section{Results}

Fourteen ( 7 men, 7 women) consecutive patients with drug refractory advanced $\mathrm{CHF}$
Table 1. Biochemical and hematologic values.

\begin{tabular}{|c|c|}
\hline Parameters & Mean \pm SD \\
\hline Glucose [mg/dL] & $133.21 \pm 71.92$ \\
\hline BUN [mg/dL] & $30.92 \pm 18.09$ \\
\hline Creatinine $[\mathrm{mg} / \mathrm{dL}]$ & $1.20 \pm 0.60$ \\
\hline Uric acid [mg/dL] & $5.22 \pm 0.99$ \\
\hline AST [U/L] & $21.35 \pm 4.71$ \\
\hline ALT [U/L] & $17.07 \pm 8.53$ \\
\hline Cholesterol [mg/dL] & $169.85 \pm 37.22$ \\
\hline $\mathrm{HDL}[\mathrm{mg} / \mathrm{dL}]$ & $39.78 \pm 14.78$ \\
\hline LDL [mg/dL] & $100.21 \pm 22.52$ \\
\hline Triglyceride [mg/dL] & $157.14 \pm 83.53$ \\
\hline Sodium [mEq/L] & $138.78 \pm 3.06$ \\
\hline Potassium [mEq/L] & $4.57 \pm 0.51$ \\
\hline Chlorine [mEq/L] & $101.07 \pm 3.54$ \\
\hline Calcium [mg/dL] & $9.13 \pm 0.37$ \\
\hline Magnesium [mg/dL] & $2.09 \pm 0.17$ \\
\hline Free T3 [pg/mL] & $2.90 \pm 0.44$ \\
\hline Free T4 [pg/mL] & $1.09 \pm 0.20$ \\
\hline TSH [ulU/mL] & $1.56 \pm 1.36$ \\
\hline Leukocytes [/uL] & $7.71 \pm 1.56$ \\
\hline Hemoglobin $[\mathrm{g} / \mathrm{dL}]$ & $12.92 \pm 1.14$ \\
\hline Hematocrit [\%] & $37.21 \pm 3.27$ \\
\hline Platelets [/uL] & $233.5 \pm 48.77$ \\
\hline $\mathrm{MCV}[\mathrm{fL}]$ & $86.03 \pm 10.03$ \\
\hline MPV [fL] & $8.79 \pm 1.07$ \\
\hline
\end{tabular}

SD — standard deviation; BUN — blood urea nitrogen; AST — aspartat aminotransferase; ALT — alanin aminotransferase; $\mathrm{HDL}$ - high density lipoprotein; LDL — low density lipoprotein; TSH - thyroid stimulating hormone; ESR - erythrocyte sedimentation rate; CRP - C-reactive protein; MCV - mean corpuscular volume; MPV — mean platelet volume

were included in the study. The mean age of the CHF patients was $53.6 \pm 9.1$ years. All patients had non-ischemic cardiomyopathy. Ten $(81 \%)$ patients were in NYHA class III, while the remaining 4 were in class IV. Six patients had diabetes mellitus. During the study all patients were receiving angiotensin-converting enzyme or angiotensin II receptor blocker-thiazide diuretics combination; beta blocker and spirinolactone. Table 1 shows biochemical and hematologic values of the patients. Table 2 shows hemodynamic and echocardiographic values, $\mathrm{NE}$ and BNP levels in pre- and post-CRT. Although systolic blood pressure, diastolic blood pressure, LV end-diastolic diameter, LV end-systolic diameter, serum $\mathrm{BNP}$, and serum NE levels significantly decreased after CRT implantation; EF and aortic distensibility significantly increased. There was no significance in the hemodynamic and echocardiographic values, $\mathrm{NE}$ and BNP levels in pre- and post-CRT between men and women (Table 3 ). 
Table 2. Hemodynamic and echocardiographic values, norepinephrine and brain natriuretic peptide levels in pre- and post- cardiac resynchronization therapy (CRT).

\begin{tabular}{|c|c|c|c|}
\hline & Pre-CRT & Post-CRT & $\mathbf{P}$ \\
\hline $\mathrm{SBP}[\mathrm{mm} \mathrm{Hg}]$ & $114.92 \pm 16.59$ & $106.92 \pm 12.79$ & 0.01 \\
\hline $\mathrm{DBP}[\mathrm{mm} \mathrm{Hg}]$ & $72.50 \pm 13.82$ & $65.00 \pm 10.44$ & 0.001 \\
\hline Heart rate $[\mathrm{bpm}]$ & $80.92 \pm 14.34$ & $82.92 \pm 15.21$ & 0.72 \\
\hline LVEDD [mm] & $6.99 \pm 1.11$ & $6.76 \pm 1.16$ & 0.001 \\
\hline LVESD [mm] & $6.03 \pm 1.13$ & $5.63 \pm 1.18$ & 0.001 \\
\hline Ejection Fraction [\%] & $23.96 \pm 3.98$ & $28.64 \pm 5.79$ & 0.001 \\
\hline AoDd $[\mathrm{mm}]$ & $3.24 \pm 0.48$ & $3.25 \pm 0.45$ & 0.72 \\
\hline AoSd $[\mathrm{mm}]$ & $3.49 \pm 0.58$ & $3.55 \pm 0.57$ & 0.11 \\
\hline Aortic distensibility $\left[\mathrm{cm}^{2} /\right.$ dyne $\left.10^{-6}\right]$ & $2.71 \pm 1.30$ & $3.39 \pm 1.37$ & 0.001 \\
\hline B-type natriuretic peptide $[\mathrm{pg} / \mathrm{ml}]$ & $409.30 \pm 312.68$ & $188.82 \pm 159.57$ & 0.001 \\
\hline Norepinephrine [nmol/L] & $34179.05 \pm 117835.34$ & $89.1091 \pm 133.7339$ & 0.01 \\
\hline
\end{tabular}

SBP — systolic blood pressure; DBP — diastolic blood pressure; LVEDD — left ventricular end-diastolic diameter; LVESD — left ventricular end-systolic diameter, AoDd — aortic diastolic diameter; AoSd — aortic systolic diameter

Table 3. Hemodynamic and echocardiographic values, norepinephrine and brain natriuretic peptide levels in pre- and post- cardiac resynchronization therapy between men and women.

\begin{tabular}{|c|c|c|c|}
\hline & Men & Women & $\mathbf{P}$ \\
\hline Age [years] & $56.8 \pm 9.7$ & $50.4 \pm 7.7$ & 0.12 \\
\hline $\mathrm{SBP}[\mathrm{mm} \mathrm{Hg}] 1$ & $113.42 \pm 17.98$ & $116.42 \pm 16.37$ & 1.00 \\
\hline $\mathrm{SBP}[\mathrm{mm} \mathrm{Hg}] 2$ & $107.57 \pm 13.79$ & $106.28 \pm 12.77$ & 0.62 \\
\hline $\mathrm{DBP}[\mathrm{mm} \mathrm{Hg}] 1$ & $65.71 \pm 13.49$ & $79.28 \pm 11.16$ & 0.09 \\
\hline $\mathrm{DBP}[\mathrm{mm} \mathrm{Hg}] 2$ & $60.42 \pm 11.48$ & $69.57 \pm 7.45$ & 0.12 \\
\hline Heart rate $[\mathrm{bpm}] 1$ & $75.71 \pm 16.89$ & $86.14 \pm 9.83$ & 0.12 \\
\hline Heart rate $[\mathrm{bpm}] 2$ & $77.00 \pm 14.03$ & $88.85 \pm 14.92$ & 0.16 \\
\hline LVEDD [mm] 1 & $7.11 \pm 1.30$ & $6.87 \pm 0.98$ & 0.90 \\
\hline LVEDD [mm] 2 & $6.85 \pm 1.39$ & $6.66 \pm 0.99$ & 0.90 \\
\hline LVESD [mm] 1 & $6.10 \pm 1.22$ & $5.97 \pm 1.13$ & 1.00 \\
\hline LVESD [mm] 2 & $5.72 \pm 1.30$ & $5.54 \pm 1.14$ & 0.90 \\
\hline Ejection Fraction [\%] 1 & $25.42 \pm 3.95$ & $22.50 \pm 3.71$ & 0.16 \\
\hline Ejection Fraction [\%] 2 & $29.57 \pm 6.90$ & $27.71 \pm 4.82$ & 0.80 \\
\hline AoDd [mm] 1 & $3.42 \pm 0.61$ & $3.06 \pm 0.21$ & 0.16 \\
\hline AoDd $[\mathrm{mm}] 2$ & $3.38 \pm 0.55$ & $3.12 \pm 0.29$ & 0.31 \\
\hline AoSd $[\mathrm{mm}] 1$ & $3.71 \pm 0.72$ & $3.27 \pm 0.29$ & 0.16 \\
\hline AoSd $[\mathrm{mm}] 2$ & $3.74 \pm 0.70$ & $3.36 \pm 0.38$ & 0.16 \\
\hline Aortic distensibility $\left[\mathrm{cm}^{2} /\right.$ dyne $\left.10^{-6}\right] 1$ & $2.63 \pm 1.12$ & $2.79 \pm 1.54$ & 1.00 \\
\hline Aortic distensibility $\left[\mathrm{cm}^{2} /\right.$ dyne $\left.10^{-6}\right] 2$ & $3.32 \pm 1.32$ & $3.47 \pm 1.51$ & 0.71 \\
\hline B-type natriuretic peptide $[\mathrm{pg} / \mathrm{ml}] 1$ & $425.29 \pm 391.31$ & $393.31 \pm 241.07$ & 1.00 \\
\hline B-type natriuretic peptide $[\mathrm{pg} / \mathrm{ml}] 2$ & $192.30 \pm 216.15$ & $185.34 \pm 91.78$ & 0.38 \\
\hline Norepinephrine [nmol/L] 1 & $65351.23 \pm 166673.23$ & $3006.8739 \pm 6103.6027$ & 0.90 \\
\hline Norepinephrine [nmol/L] 2 & $58.7161 \pm 67.5731$ & $119.5020 \pm 178.9659$ & 0.62 \\
\hline
\end{tabular}

SBP — systolic blood pressure; DBP — diastolic blood pressure; LVEDD — left ventricular end-diastolic diameter; LVESD - left ventricular end-systolic diameter, AoDd - aortic diastolic diameter; AoSd - aortic systolic diameter; 1 - pre-cardiac resynchronization therapy; 2 - post-cardiac resynchronization therapy 


\section{Discussion}

The current study examined the acute effects of CRT on serum NE and arterial distensibility in patients with severe CHF. The major findings of this study is that after implantation of CRT serum $\mathrm{NE}$ levels decrease and the arterial distensibility in patients with $\mathrm{CHF}$ in acute period improves.

With the progression of CHF, several neurohumoral systems, including the renin-angiotensinaldosterone system, sympathetic nervous system and natriuretic peptides such as BNP are activated [14]. BNP release is stimulated by stretching of the atrial tissue and by volume-loading of the circulation, as in our study. Therefore, BNP levels correlate with the severity of CHF and predict prognosis of CHF patients [15]. Greater levels of BNP prior to implantation predict a positive response to CRT in HF [16]. Levels of atrial natriuretic peptide and BNP consistently decrease after a 3-month CRT compared with baseline $[17,18]$.

Sympathetic activation causes an increase in heart rate and inotropy via the release of $\mathrm{NE}$ acting primarily upon beta1-adrenoceptors, during CHF serves as an important compensatory mechanism, but it is also a precipitating factor in progressive CHF. Increased sympathetic tone in CHF leads to substantially elevated plasma NE level and impaired reuptake and storage function of this primary neurohormonal transmitter at the presynaptic level, contributing to disease progression and an unfavorable prognosis [19, 20]. Many studies demonstrated that CRT improves in symptoms and exercise capacity in patients with $\mathrm{CHF}$, as in our study [21-24]. These beneficial clinical effects were accompanied by evidence of reverse ventricular remodeling, which included improvements in biventricular mechanical resynchronization, mitral regurgitation, a significant increase in LVEF and significant reductions in LV end-systolic volume, $\mathrm{LV}$ end-diastolic volume and following CRT, as in our study [21, 22, 25]. The most important finding of our study was the decrease of cardiac sympathetic activation, assessed on the basis of serum NE levels, after CRT. Cha et al. [25] found that clinical and cardiac functional improvements were paralleled by significant improvements in $\mathrm{NE}$ reuptake function as measured by metaiodobenzylguanidine $\left({ }^{123} \mathrm{I}-\mathrm{MIBG}\right)$, an NE analog, uptake and retention after CRT for advanced cardiomyopathy. Also, CRT inhibits arterial baroreflex mediated sympathoexcitation occurring in CHF, and, there- fore, reduces adrenergic nerve outflow measured by microneurography $[26,27]$.

In this study, we found that arterial stiffness and serum NE levels are decreased after CRT therapy. Arterial distensibility plays a great clinical role in describing patients at high cardiovascular risk $[4,28]$. It is an important mechanical property as it is related to the impedance of blood vessels and in turn to the pulsatile afterload that is presented to the LV [29]. Several studies have shown that arterial distensibility depends on variations in blood pressure [4, 28], as well as sympathetic stimulation [30-32]. Activation of the sympathetic nervous system has been shown to reduce distensibility of small- and medium-size arteries in animals [30,31]. Also, one study demonstrated that increases in sympathetic stimulation are associated with the reduction of radial artery distensibility in humans [33]. The sympathetic nervous system exerts a marked tonic restraint on large arterial distensibility, and this restraint involves large arteries with a predominant elastic structure and arteries with a predominant muscle structure. The decrease in aortic distensibility may increase the impedance to LVEF and, thus, may reduce the coronary blood flow and aggravating myocardial ischemia.

\section{Limitations of the study}

The medications used during the study may affect echocardiographic measurements, and circulating plasma catecholamine levels. Concomitant use of beta blocker and angiotensin converting enzyme/angiotensin II receptor inhibitor may suppress elevated NE level or attenuate the increase in $\mathrm{NE}$ as previously reported [34,35].

\section{Conclusions}

In conclusion, after implantation of CRT activation of sympathetic activity decreases and the arterial distensibility in patients with CHF improves.

\section{Acknowledgments}

We acknowledge The Scientific and Technological Research Council of Turkey (TUBITAK) and Deutsche Forschungsgemeinschaft (DFG). Also, we acknowledge the valuable doctors, Professor Dietmar Bänsch and Ibrahim Akin, Department of Cardiology (Cardiac Electrophysiology Division), Medical Faculty of University Rostock.

\section{Conflict of interest: none declared}




\section{References}

1. Hunt SA, Abraham WT, Chin MH, et al: 2009 Focused Update Incorporated Into the ACC/AHA 2005 Guidelines for the Diagnosis and Management of Heart Failure in Adults A Report of the American College of Cardiology Foundation/American Heart Association Task Force on Practice Guidelines: Developed in collaboration with the International Society for Heart and Lung Transplantation. Circulation, 2009; 119: e391-e479.

2. Epstein AE, DiMarco JP, Ellenbogen KA et al. ACC/AHA/HRS 2008 Guidelines for Device-Based Therapy of Cardiac Rhythm Abnormalities: a report of the American College of Cardiology/ /American Heart Association Task Force on Practice Guidelines (Writing Committee to Revise the ACC/AHA/NASPE 2002 Guideline Update for Implantation of Cardiac Pacemakers and Antiarrhythmia Devices): Developed in collaboration with the American Association for Thoracic Surgery and Society of Thoracic Surgeons. Circulation, 2008; 117: e350-e408.

3. Gullestad L, Aukrust P. Review of trials in chronic heart failure showing broad-spectrum anti-inflammatory approaches. Am J Cardiol, 2005; 95: 17C-23C.

4. Asmar R, Benetos A, Topouchian J et al. Assessment of arterial distensibility by automatic pulse wave velocity measurement. Validation and clinical application studies. Hypertension, 1995; 26: 485-490.

5. Stefanadis C, Wooley CF, Bush CA et al. Aortic distensibility abnormalities in coronary artery disease. Am J Cardiol, 1987; 59: 1300-1304.

6. Hirai T, Sasayama S, Kawasaki T et al. Stiffness of systemic arteries in patients with myocardial infarction. A non-invasive method to predict coronary atherosclerosis. Circulation, 1989; 80: 78-86.

7. Rubaj A, Ruciński P, Rejdak K et al. Biventricular versus right ventricular pacing decreases immune activation and augments nitric oxide production in patients with chronic heart failure. Eur J Heart Fail, 2006; 8: 615-620.

8. Boriani G, Regoli F, Saporito D et al. Neurohormones and infammatory mediators in patients with heart failure undergoing cardiac resynchronization therapy: Time courses and prediction of response. Peptides, 2006; 27: 1776-1786.

9. Theodorakis GN, Flevari P, Kroupis $\mathrm{C}$ et al. Anti-infammatory effects of cardiac resynchronization therapy in patients with chronic heart failure. Pacing Clin Electrophysiol, 2006; 29: 255-261.

10. Pearlman AS, Gardin JM, Martin RP et al. Guidelines for optimal physician training in echocardiography. Recommendations of the American Society of Echocardiography Committee for Physician Training in Echocardiography. Am J Cardiol, 1987; 60: 158-163.

11. Stefanadis C, Stratos C, Boudoulas H et al. Distensibility of the ascending aorta: comparison of invasive and noninvasive techniques in healthy men and in men with coronary artery disease. Eur Heart J, 1990; 11: 990-996.

12. Stefanadis C, Stratos C, Vlachopoulos C et al. Pressure-diameter relation of the human aorta: a new method of determination by the application of a special ultrasonic dimension catheter. Circulation, 1995; 92: 2210-2219.

13. Lilli A, Ricciardi G, Porciani MC et al. Cardiac resynchronization therapy: gender related differences in left ventricular reverse remodeling. Pacing Clin Electrophysiol, 2007; 30: 1349-1355.

14. Toischer K, Kögler H, Tenderich $\mathrm{G}$ et al. Elevated afterload, neuroendocrine stimulation, and human heart failure increase BNP levels and inhibit preload-dependent SERCA upregulation. Circ Heart Fail, 2008; 1: 265-271.

15. Auerbach SR, Richmond ME, Lamour JM et al. BNP levels predict outcome in pediatric heart failure patients: post hoc analysis of the Pediatric Carvedilol Trial. Circ Heart Fail, 2010; 3: 606-611.

16. Lellouche N, De Diego C, Cesario DA et al. Usefulness of preimplantation B-type natriuretic peptide level for predicting response to cardiac resynchronization therapy. Am J Cardiol, 2007; 99: 242-246.

17. Uretsky BF, Thygesen K, Daubert JC et al. Predictors of mortality from pump failure and sudden cardiac death in patients with systolic heart failure and left ventricular dyssynchrony: Results of the CARE-HF trial. J Card Fail, 2008; 14: 670-675.

18. Yu CM, Bleeker GB, Fung JW et al. Left ventricular reverse remodeling but not clinical improvement predicts long-term survival after cardiac resynchronization therapy. Circulation, 2005;112: 1580-1586.

19. Cohn JN, Levine TB, Olivari MT et al. Plasma norepinephrine as a guide to prognosis in patients with chronic congestive heart failure. N Engl J Med, 1984; 311: 819-823.

20. Kaye D, Lefkovits J, Jennings G et al. Adverse consequences of high sympathetic nervous activity in the failing human heart. J Am Coll Cardiol, 1995; 26: 1257-1263.

21. Abraham WT, Fisher WG, Smith AL et al.; MIRACLE Study Group. Multicenter InSync Randomized Clinical Evaluation. Cardiac resynchronization in chronic heart failure. N Engl J Med, 2002; 346: 1845-1853.

22. Cazeau S, Leclercq C, Lavergne T et al.; Multisite Stimulation in Cardiomyopathies (MUSTIC) Study Investigators. Effects of multisite biventricular pacing in patients with heart failure and intraventricular conduction delay. N Engl J Med, 2001; 344: 873-880.

23. Tang AS, Wells GA, Talajic M et al.; Resynchronization-Defibrillation for Ambulatory Heart Failure Trial Investigators. Cardiacresynchronization therapy for mild-to-moderate heart failure. N Engl J Med, 2010; 363: 2385-2395.

24. Linde C, Abraham WT, Gold MR et al., REVERSE Study Group. Cardiac resynchronization therapy in asymptomatic or mildly symptomatic heart failure patients in relation to etiology: results from the REVERSE (REsynchronization reVErses Remodeling in Systolic Left vEntricular Dysfunction) study. J Am Coll Cardiol, 2010; 56: 1826-1831.

25. Cha YM, Oh J, Miyazaki C et al. Cardiac resynchronization therapy upregulates cardiac autonomic control. J Cardiovasc Electrophysiol, 2008; 19: 1045-1052.

26. Grassi G, Vincenti A, Brambilla R et al: Sustained sympathoinhibitory effects of cardiac resynchronization therapy in severe heart failure. Hypertension, 2004; 44: 727-731.

27. Hamdan MH, Zagrodzky JD, Joglar JA et al. Biventricular pacing decreases sympathetic activity compared with right ventricular pacing in patients with depressed ejection fraction. Circulation, 2000; 102: 1027-1032.

28. Yildiz M. Arterial distensibility in chronic inflammatory rheumatic disorders. Open Cardiovasc Med J, 2010; 4: 83-88.

29. Mackenzie S, Wilkinson IB, Cockcroft JR. Assessment of arterial stiffness in clinical practice. Q J Med, 2002; 95: 67-74.

30. Cox RH. Effects of norepinephrine on mechanics of arteries in vitro. Am J Physiol, 1976; 231: 420-425.

31. Cox RH, Bagshaw RJ: Effects of pulsations on carotid sinus control of regional arterial hemodynamics. Am J Physiol, 1980; 238: H182-H190.

32. Yildiz M, Sahin B, Sahin A. Acute effects of oral melatonin administration on arterial distensibility, as determined by carotidfemoral pulse wave velocity, in healthy young men. Exp Clin Cardiol, 2006; 11: 311-313.

33. Boutouyrie P, Lacolley P, Girerd X et al. Sympathetic activation decreases medium sized arterial compliance in humans. Am J Physiol, 1994; 267: H1368-H1377.

34. Latini R, Masson S, Anand I et al.; Valsartan Heart Failure Trial Investigators. Effects of Valsartan on circulating brain natriuretic peptide and norepinephrine in symptomatic chronic heart failure: The Valsartan heart failure trial (Val-HeFT). Circulation, 2002; 106: 2454-2458.

35. Azevedo ER, Kubo T, Mak S et al. Nonselective versus selective betaadrenergic receptor blockade in congestive heart failure: Differential effects on sympathetic activity. Circulation, 2001;104: 2194-2199. 